\title{
Premier inventaire des macro-invertébrés du fleuve Niger à Niamey comme bioindicateurs de la pollution urbaine et industrielle
}

\section{First inventory of macroinvertebrates of River Niger in Niamey as bioindicators of urban and industrial pollution}

\author{
B. Alhou(1), Y. Issiaka (2), A. Awaiss ${ }^{(3)}$, J.-C. Micha(4) \\ (1) Département des Sciences de la Vie et de la Terre, École Normale Supérieure, Université Abdou Moumouni \\ de Niamey, BP 10963, Niger \\ (2) Département Génie Rural et Eaux et Forêts, Faculté d'Agronomie et des Sciences de l'Environnement, \\ Université de Maradi BP 465, Maradi, Niger \\ youssoufa_maiga@yahoo.fr \\ (3) Département des Eaux et Forêts, Faculté d'Agronomie, Université ABDOU Moumouni de Niamey, \\ BP 10 960, Niger \\ aawaiss_wwf@wwfafrica.org \\ (4) FUNDP, Département de Biologie, Unité de Recherche en Biologie des Organismes, 61 rue de Bruxelles, \\ 5000 Namur, Belgique \\ jean-claude.micha@fundp.ac.be
}

Résumé - L'approche biologique, basée sur la distribution des peuplements de macro-invertébrés le long du fleuve Niger à Niamey, permet une bonne caractérisation biologique des stations d'échantillonnage. L'échantillonnage des macro-invertébrés à chacune de ces stations est effectué grâce au filet troubleau, aux substrats artificiels et à la jacinthe d'eau. Cette approche montre l'évolution de la richesse et de la diversité taxonomique le long des stations. Ainsi, la richesse et la diversité taxonomique diminuent en passant de l'amont à l'aval des points de rejets. Les stations de référence et les stations en aval éloigné des rejets présentent une diversité plus satisfaisante comparativement aux stations en aval immédiat des rejets. II ressort de l'ordination des taxons que les stations en amont et en aval éloigné des rejets sont les moins polluées et abritent des taxons qui sont sensibles à la pollution comme Thraulus sp., Elassoneuria sp., Afronurus sp., Centroptiloides sp., Adenophlebia sp., Dipseudopsis $s p$.et Neoperla $s p$. Les stations en aval immédiat de la brasserie du Niger et de l'abattoir paraissent être les plus polluées avec la présence des taxons comme Chironomus gr. plumosus, Syrphidae et Culicidae. Le changement dans la composition des assemblages taxonomiques, l'indice de diversité de Shannon-Weaver et l'abondance des taxons sont des indicateurs d'une modification de la qualité de l'eau et de l'habitat en passant de l'amont à l'aval des points de rejets de la ville de Niamey.

Mots-clés - fleuve Niger, macro-invertébrés, Niamey, qualité de l'eau, Niger 


\begin{abstract}
The biological approach, based on the distribution of macroinvertebrate communities along the River Niger in Niamey, allows a good characterization of biological sampling stations. Macroinvertebrate sampling at each of these stations is done through kicknet, the artificial substrates and water hyacinth. This approach shows the evolution of the taxonomic richness and diversity along the stations. Thus, the taxonomic richness and diversity decrease upstream to downstream discharge points. Reference stations and stations downstream have more diversity compared to stations immediately downstream waste water discharge. It appears from the ordination of taxa that stations upstream and downstream discharges are far less polluted and contain taxa that are sensitive to pollution as Thraulus $s p$. Elassoneuria sp., Afronurus sp., Centroptiloides sp., Adenophlebia sp., Dipseudopsis sp. et Neoperla $s p$. Stations immediately downstream of the brewery and slaughter seem to be more polluted with the presence of taxa such as Chironomus gr. plumosus, Syrphidae and Culicidae. The change in the taxonomic community, diversity index of Shannon-Weaver and taxa abundance are indicators of a change in water quality and habitat from upstream to downstream of the discharge points of the city of Niamey.
\end{abstract}

Key words - River Niger, macroinvertebrates, Niamey, Niger, water quality

\section{INTRODUCTION}

L'utilisation des macro-invertébrés dans l'étude de la qualité des eaux est devenue très fréquente et diversifiée, ce qui explique l'explosion de nombreuses méthodes utilisées actuellement pour évaluer l'état des écosystèmes aquatiques. Ces méthodes portent entre autres sur l'étude de la structure et de la composition des macro-invertébrés (Tumwesigye et al., 2000 ; Lomond \& Colbo, 2000), l'utilisation des indices biotiques (Solimini et al., 2000 ; Lafont et al., 1991), l'utilisation des traits biologiques (Mondy et al., 2012; Vanden Bossche \& Usseglio-Polatera, 2005 ; Statzner et al., 2004 ; Gayraud et al., 2003 ; UsseglioPolatera et al., 2000) et l'utilisation des analyses multivariées qui mettent en relation les facteurs environnementaux et les communautés de macro-inverébrés (Floury et al., 2013 ; Piscart et al., 2005 ; Sarkar et al., 2005 ; Shivoga, 2001 ; Bauernfeind \& Moog, 2000 ; Lancaster, 1999).
Cette publication porte sur l'inventaire de la diversité taxonomique des macro-invertébrés du fleuve Niger à Niamey à différentes stations à partir de trois techniques d'échantillonnage (filet troubleau, substrats artificiels et jacinthe d'eau).

L'objectif principal de cette publication est d'utiliser les macro-invertébrés pour mettre en évidence les variations biologiques et écologiques le long du fleuve Niger à proximité de Niamey qui rejette toutes ses eaux usées dans ce fleuve.

\section{MATÉRIELS ET MÉTHODES}

\section{Présentation de la zone d'étude}

Cette étude est entreprise le long du fleuve Niger à Niamey. Niamey est située à $2,01^{\circ}$ Est, $13,57^{\circ}$ Nord en République du Niger. Les stations étudiées se situent à $186 \mathrm{~m}$ d'altitude en moyenne. 
Le bassin du fleuve Niger appartient à un vaste espace géographique hétérogène composé de dix pays (Bénin, Burkina Faso, Cameroun, Côte d'Ivoire, Guinée, Mali, Niger, Nigeria, Tchad et Algérie). II draine une superficie d'environ $2120000 \mathrm{~km}^{2}$. De part cette appartenance géographique, il présente des caractéristiques physiographiques, socio-économiques et écologiques variables. Le bassin du Niger constitue par sa taille le deuxième système pluvial au monde.

Son cours d'eau principal, le fleuve Niger prend sa source dans les montagnes du Fouta Djallon en Guinée à une altitude de $800 \mathrm{~m}$ environ avec une direction Nord-Est. II passe ainsi par la bordure du Sahara après la traversée au Mali du delta intérieur, vaste plaine inondable d'une superficie de $80000 \mathrm{~km}^{2}$ qui dissipe par absorption et évaporation une importante partie de son potentiel hydraulique. II est en longueur le troisième fleuve d'Afrique après le Nil et le Congo et le neuvième au monde avec $4200 \mathrm{~km}$ dont $550 \mathrm{~km}$ en territoire nigérien. Le tronçon étudié représente environ $17 \mathrm{~km}$. Le débit moyen du fleuve Niger à Niamey durant la période d'étude est de $264 \mathrm{~m}^{3} \mathrm{~s}^{-1}$ en étiage et $1309 \mathrm{~m}^{3} \mathrm{~s}^{-1}$ pendant la crue. Les prélèvements sont effectués à une profondeur moyenne de $0,80 \mathrm{~m}$.

\section{Échantillonnage}

Trois méthodes d'échantillonnage ont été utilisées ; il s'agit des substrats artificiels, du filet troubleau et de la jacinthe d'eau. Le choix de ces méthodes est basé essentiellement sur le régime hydrologique du fleuve Niger (étiage et hautes eaux) qui est un facteur déterminant dans l'applicabilité et l'efficacité de chacune d'elles. En étiage, période correspondant à des eaux peu profondes et à des courants faibles, les substrats artificiels et le filet troubleau ont servi à la récolte des macro-invertébrés tandis qu'en hautes eaux, les macro-invertébrés ont été récoltés dans le système racinaire de la jacinthe d'eau à cause de la profondeur des eaux qui n'est pas favorable à l'utilisation des deux premières méthodes. L'utilisation non conjointe de ces méthodes limite leur comparaison, ce qui d'ailleurs n'est pas l'objectif visé par ce travail.

Les 10 stations échantillonnées (TON 1, HNN, UAM, GH, TAN, ME, ABA, BRA, ENI et SA 2) sont représentées sur la figure 1.

\section{Substrats artificiels}

L'utilisation des substrats artificiels permet une standardisation de l'échantillonnage en offrant à toutes les stations un micro-habitat uniforme pour les macro-invertébrés avec une possibilité de comparaison de celles-ci.

Dans le cadre de cette étude, les substrats, d'une longueur de $48 \mathrm{~cm}$, une largeur de $38 \mathrm{~cm}$ et une hauteur de $10 \mathrm{~cm}$, ont été conçus avec des pierres de taille plus ou moins égale $(4$ à $8 \mathrm{~cm}$ ) enfermées dans du grillage galvanisé.

\section{Procédure et fréquence d'échantillonnage}

II est généralement recommandé trois à six substrats artificiels par station 


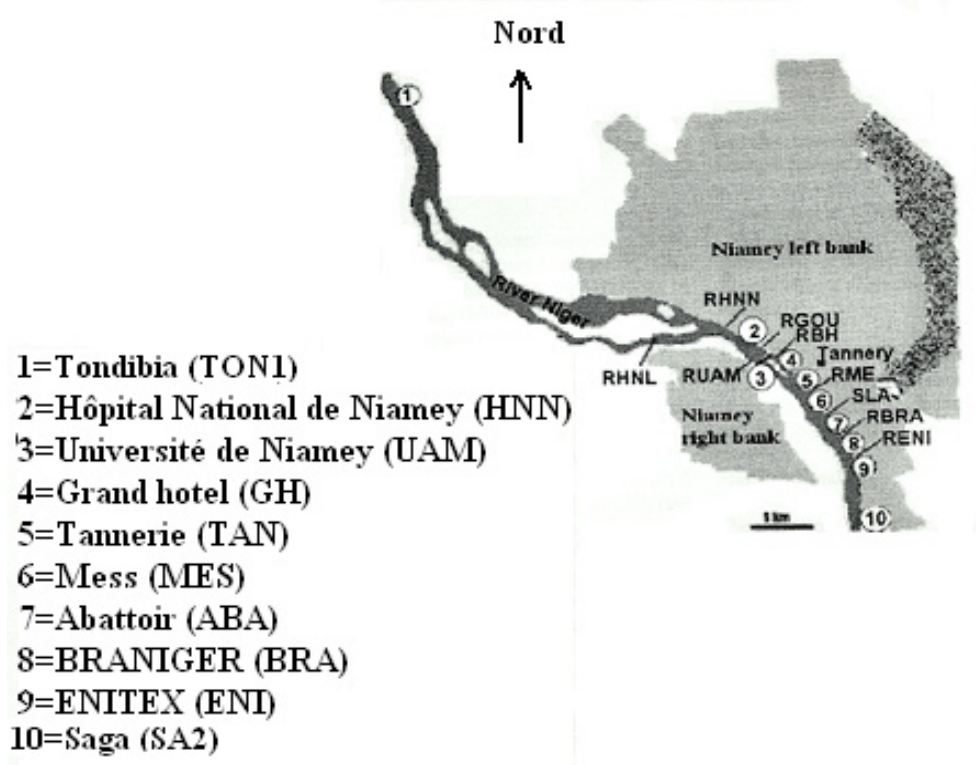

Fig. 1. Carte de localisation des sites d'échantillonnage le long du fleuve Niger à Niamey (zone en gris). (RHNL = rejets de l'hôpital national de Lamordé ; RHNN = rejets de l'hôpital national de Niamey ; RUAM = rejets de l'Université Abdou Moumouni ; RGOU = rejets domestiques de Goutiyena ; RGH = rejets domestiques du Grand hôtel ; RME = rejets domestiques de Mess ; RABA = rejets de l'abattoir ; RBRA = rejets de la brasserie du Niger; RENI = rejets de l'entreprise Nigérienne de textile.)

Fig. 1. Map of sampling sites along the River Niger in Niamey (gray area). (RHNL = discharges from the national hospital of Lamordé; RHNN = discharges from the national hospital of Niamey ; RUAM = discharges from the Abdou Moumouni University; RGOU = domestic discharges from the Goutiyena; $\mathrm{RBH}=$ domestic discharges from the Big hotel; $\mathrm{RME}=$ domestic discharges from the Mess; RABA = discharges from the slaughter; RBRA = discharges from the Niger brewery; RENI = discharges from the Niger textile company.)

(Pelletier, 2002). Dans le cadre de ce travail, quatre substrats artificiels ont été utilisés par station de mai à juin 2004 et huit d'avril à mai 2005 pour parer à la perte de substrats constatée en 2004 soit deux campagnes au total. Ils sont posés au fond sur deux rangées parallèles en maintenant entre substrats une distance d'un mètre. Les substrats sont laissés dans l'eau pendant six semaines avant d'être relevés. Cette durée correspond à la période jugée minimale pour permettre une colonisation stable des substrats par les macro-invertébrés.

\section{Collecte des substrats}

Les substrats sont soigneusement retirés de l'eau en utilisant un filet moustiquaire de petites mailles $(250 \mu \mathrm{m})$ et de couleur blanche pour éventuellement récupérer les organismes qui chutent. Chaque substrat est immédiatement transféré dans un 
récipient en plastique contenant de l'eau du fleuve. Les rochers et la grille sont rigoureusement débarrassés des macro-invertébrés qu'ils supportent en utilisant un scalpel. Le contenu du récipient est conservé dans des bocaux contenant du formol $10 \%$ pour un tri au laboratoire.

\section{Difficultés rencontrées dans l'application de cette méthode}

La principale difficulté liée à l'utilisation de cette méthode sur le fleuve Niger à Niamey est la présence humaine qui occasionne souvent la perte des substrats. Le taux de perte des substrats est de $20 \%$ en 2004 et $27 \%$ en 2005.

\section{Filet troubleau}

Un filet troubleau à mailles de $500 \mu \mathrm{m}$ avec une ouverture carrée de $21 \mathrm{~cm}$ de côté, monté sur une manche en acier de 1,5 $\mathrm{m}$ de long est utilisé sur les rives en étiage. Lors de l'échantillonnage, l'ouverture du filet est placée à contre-courant et le filet est traîné légèrement sur le fond le long d'un transect de $100 \mathrm{~m}$ de long en passant par le maximum d'habitats possibles. Le filet est ensuite relevé et le contenu bien concentré est reversé dans des bocaux contenant du formol $10 \%$. Le tri est effectué plus tard au laboratoire.

La superficie échantillonnée est de $100 \mathrm{~m} \times 0,21 \mathrm{~m}$ soit $21 \mathrm{~m}^{2}$.

Cette méthode a été appliquée une fois par mois en mai et juin 2005 puis en mars 2006 soit trois campagnes au total.

\section{Jacinthe d'eau}

Le choix porté sur ce macrophyte pour la récolte des macro-invertébrés est lié à son abondance dans les zones calmes et à la densité de son système racinaire qui peut être un habitat favorable pour ces êtres vivants aquatiques (Viljoen et al., 2001).

La jacinthe d'eau, Eichornia crassipes, est une plante aquatique présente dans plusieurs pays du monde à température supérieure à $15^{\circ} \mathrm{C}$. Originaire du bassin du fleuve Amazone, la jacinthe d'eau est actuellement répandue sur tous les continents. Sa longueur peut atteindre $20 \mathrm{~cm}$ et sa largeur 5 à $15 \mathrm{~cm}$. Elle a la facilité de pousser dans des conditions environnementales et nutritives diverses. Néanmoins, sa prolifération est beaucoup plus rapide quand l'eau est chargée en polluants (matières en suspension, phosphates, nitrates). Cette prolifération se fait dans les baies et les abords des cours d'eau où le courant est moins fort. Elle se reproduit principalement par clonage mais également à partir des graines qui jouent un rôle dans sa survie et la colonisation des plans d'eau.

La jacinthe d'eau affecte non seulement la santé des riverains en favorisant la prolifération des maladies telles que la bilharziose et la malaria mais également l'état de l'écosystème aquatique en réduisant sa biodiversité et en altérant la qualité chimique des eaux. Elle constitue de ce fait une espèce nuisible pour les riverains et l'écosystème aquatique. 


\section{Récolte des macro-invertébrés}

Elle est effectuée sur les racines de jacinthe d'eau quatre mois après leur stabilisation en saison des pluies. Seule la partie immergée de la plante est prélevée. L'échantillonnage de la jacinthe d'eau est réalisé en différents points de la station selon un transect longitudinal. Pour ce faire, un filet troubleau à maille de $500 \mu \mathrm{m}$ est placé en dessous du système racinaire qui est directement coupé, dans celui-ci, avec une paire de ciseaux.

Un échantillon composé d'au moins 10 prélèvements est constitué en février 2005 et 2006 à chaque station.

Un prélèvement correspond à une biomasse racinaire sèche de $10 \mathrm{~g}$ environ. Tous les prélèvements sont conservés dans des bocaux contenant du formol $10 \%$ puis ensuite triés au laboratoire. La biomasse racinaire est déterminée après plusieurs jours de séchage à l'étuve à $60{ }^{\circ} \mathrm{C}$ (Dejoux, 1991) jusqu'à poids constant et pesage direct sur une balance Mettler AK 160 de précision $0,1 \mathrm{mg}$.

\section{Tri et conservation des échantillons}

Les échantillons sont triés au laboratoire sur une colonne de tamis de mailles $5 \mathrm{~mm}, 1 \mathrm{~mm}$ et $0,4 \mathrm{~mm}$. Les organismes directement visibles sont retirés des tamis. Le contenu de chaque tamis est ensuite observé à la loupe binoculaire puis mis en suspension dans l'eau pour éventuellement reprendre le reste des organismes en suspension. Les organismes triés sont conservés au formol $4 \%$ avant leur identification.

\section{Identification des taxons}

L'identification des taxons est effectuée principalement à l'aide de la clé de Durand \& Lévêque $(1980,1981)$ et grâce à l'appui de certains spécialistes (Boudewijn Goddeeris pour les Chironomidae; Charles Verstraeten pour les Coléoptères et Henri Dumont pour les odonates). La détermination est effectuée à la loupe binoculaire Nachet NS 30 en lumière normale et dans certains cas nécessitant des préparations au microscope.

\section{Traitement des données}

L'analyse des données porte sur la structure des peuplements de macroinvertébrés notamment la richesse taxonomique, la diversité et l'équitabilité au sein des stations échantillonnées. La diversité est déterminée grâce à l'indice de Shannon-Weaver de même que l'équitabilité.

Cet indice est exprimé par la formule suivante (Quinn \& Hickey, 1990) :

$$
H=-\sum_{i}^{S}(P i)\left(\log _{2} P i\right)
$$

$H=$ indice de diversité ; $S=$ nombre de taxons ; $P i=$ proportion du taxon $i$ dans l'échantillon.

Il est subdivisé en trois classes de qualité des eaux (Evrard, 1996) :

$2 \leq H$ pour une eau propre ;

$1<H<2$ pour une eau modérément polluée ;

$H \leq 1$ pour une eau polluée.

L'indice d'équitabilité de Shannon est calculé grâce à la formule cidessous et permet d'apprécier la 
distribution des différents taxons dans les stations échantillonnées:

$$
E=\frac{H}{\log _{2}(S)}
$$

$E=$ équitabilité ; $H=$ indice de diversité de Shannon-Weaver et $S=$ nombre de taxa.

L'indice de similarité de Jaccard (Legendre \& Legendre, 1998 ; Angelier, 2000) de formule $I S=\frac{N_{c} \times 100}{N_{a}+N_{b}-N_{c}}$.

Cette formule est utilisée en vue de comparer les différents types d'échantillonnage sur la base de la présence/ absence des taxons. IS = indice de Jaccard ; $N c=$ nombre de taxons communs aux deux stations; $N_{a}$ et $N_{b}=$ nombre total de taxons dans respectivement la première et la deuxième station.

La distance de Bray-Curtis de formule $D=\frac{\sum_{j=1}^{p}\left|y_{1 j}-y_{2 j}\right|}{\sum_{j=1}^{p}\left(y_{1 j}+y_{2 j}\right)}=1-\frac{2 W}{A+B}$ (Legendre \& Legendre, 1998) est calculée pour classer les stations en fonction de leur abondance taxonomique par technique de prélèvement. $D=$ distance de Bray-Curtis, $y_{1 j}=$ nombre de taxons $j$ dans la première station, $y_{2 j}=$ nombre de taxons $j$ dans la deuxième station, $W=$ somme des abondances minimales des différents taxons entre les deux stations, $A=$ somme des abondances de tous les taxons dans la première station et $B=$ somme des abondances de tous les taxons dans la deuxième station. La distance de BrayCurtis appelée parfois distance de Sorensen, est une méthode normalisée fréquemment utilisée en botanique, en écologie et en science environnementale. Sa valeur est comprise entre 0 (nette ressemblance) et 1 (forte dissemblance). Elle est non dépendante de la double absence des espèces dans les échantillons.

Le logiciel "R" est utilisé pour calculer l'indice de similarité de Jaccard et la distance de Bray-Curtis (R Development Core Team, 2007).

Le regroupement des objets (stations/techniques de prélèvement) est effectué sur la moyenne pondérée des groupes associés.

L'analyse factorielle des correspondances (AFC) est utilisée pour déterminer les différentes communautés de macro-invertétébrés le long du fleuve Niger à Niamey. L'analyse factorielle de correspondance est appliquée sur l'abondance des taxons après une transformation logarithmique de type LOG $(X+1)$. L'option de supprimer le poids des taxons rares est appliquée. Dans le cas de la jacinthe d'eau, l'abondance des taxons est exprimée en nombre d'individus par $10 \mathrm{~g}$ de biomasse racinaire sur la jacinthe d'eau. Dans le cas des substrats artificiels en nombre d'individus par substrat artificiel (en divisant l'abondance de chaque taxon dans un échantillon par le nombre de substrats artificiels de l'échantillon). Les abondances sont exprimées de cette manière pour avoir le même effort d'échantillonnage à chaque station.

La représentation graphique de certains résultats ne tient pas compte des stations HNN et UAM dans lesquelles les prélèvements au filet troubleau et sur la jacinthe d'eau n'étaient pas possibles. 
Le logiciel utilisé pour l'analyse factorielle de correspondance est CANOCO version 4.02 (Ter Braak \& Smilauer, 1999). Statistica Kernel version 5.1 est utilisé dans le calcul des corrélations et pour tracer les clusters (Statsoft France, 1997). SigmaPlot 9.0 est utilisé pour tracer les ordinations dans le plan formé par les axes de I'AFC. Les graphiques sont ensuite retravaillés dans CANVAS version 6.0.

\section{RÉSULTATS}

Les résultats présentent la liste taxonomique, la structure et la distribution des communautés de macroinvertébrés.

\section{Liste des taxons récoltés}

Le tableau I donne la liste complète de tous les taxons récoltés pendant cette étude.

\section{Structure des communautés}

La structure des communautés est caractérisée par la distribution de la richesse et de la diversité taxonomique le long des stations échantillonnées.

\section{Richesse taxonomique}

Au total 67108 organismes appartenant à 49 familles ont été récoltés. La famille des Thiaridae (gastéropodes) est la plus abondante avec $61 \%$ d'individus suivie des Chironomidae (diptères) avec $17 \%$, des Huridinés (annélides) avec $6 \%$, des Dytiscidae (coléoptères) avec $4 \%$ et autres (12\%). Les individus appartiennent à 83 taxons dont $82 \%$ appartenant à la classe des insectes, $11 \%$ aux mollusques, $4 \%$ aux annélides, $2 \%$ aux crustacés et $1 \%$ aux némathelminthes. La famille des Chironomidae compte le plus grand nombre de taxons (20) suivie des Notonectidae (4), des Hydrophilidae, des Baetidae et des Huridinés avec chacun 3 taxons. Les odonates, les trichoptères, les éphéméroptères et les plécoptères qui sont généralement cités parmi les groupes sensibles à la pollution (Muli \& Mavuti, 2001) totalisent 20 taxons, soit $24 \%$ de la richesse taxonomique, tandis que les Chironomidae et les mollusques considérés comme des groupes indifférents à la pollution totalisent 28 taxons, soit $34 \%$. II faut également signaler la présence des Syrphidae qui est une famille de diptère caractéristique des milieux fortement pollués. Les individus de cette famille représentent $0,06 \%$ de l'effectif total des macro-invertébrés.

La richesse taxonomique des stations selon le type de prélèvement varie de 25 à 39 taxons sur la jacinthe d'eau, 9 à 35 taxons au filet troubleau et 4 à 30 taxons sur les substrats artificiels (Fig. 2).

De manière absolue, la richesse taxonomique est plus élevée, par station, dans le système racinaire de la jacinthe d'eau comparée au filet troubleau et aux substrats artificiels.

Ces deux dernières techniques présentent une richesse taxonomique beaucoup plus semblable alors que dans le système racinaire de la jacinthe d'eau, elle est beaucoup plus proche de la richesse taxonomique totale. 
Tableau I. Liste des taxons récoltés.

Table I : List of taxa collected.

\begin{tabular}{|l|}
\hline Nemathelminthes (Nem) \\
\hline Annelides \\
\hline Hirudinés 1 (Hir 1) \\
\hline Hirudinés 2 (Hir 2) \\
\hline Hirudinés 3 (Hir 3) \\
\hline Mollusques \\
\hline Pilidae \\
\hline Lanistes sp. (Lan) \\
\hline Pila sp. (Pil) \\
\hline Sphaeridae \\
\hline Sphaerium sp. (Sph) \\
\hline Ancylidae \\
\hline Burnupia sp. (Bur) \\
\hline Planorbidae \\
\hline Biomphalaria sp. (Bio) \\
\hline Thiaridae \\
\hline Melania sp. (Mel) \\
\hline Lymnaeidae \\
\hline Lymnaea sp. (Lym) \\
\hline Mutelidae \\
\hline Aspatharia sp. (Asp) \\
\hline Bulinidae \\
\hline Bulinus sp. (Bul) \\
\hline Crustacés \\
\hline Atyidae \\
\hline Caridina sp. (Car) \\
\hline Potamidae \\
\hline Potamonautes sp. (Pot) \\
\hline Ephéméroptères \\
\hline Leptophlebiidae \\
\hline Adenophlebia sp. (Ade) \\
\hline Thraulus sp. (Thr) \\
\hline Caenidae \\
\hline
\end{tabular}

\begin{tabular}{|l|}
\hline Caenis sp. (Cae) \\
\hline Baetidae \\
\hline Baetis sp. (Bae) \\
\hline Centroptiloides sp. (Cen) \\
\hline Cloeon sp. (Clo) \\
\hline Heptageniidae \\
\hline Afronurus sp. (Afr) \\
\hline Oligoneuriidae \\
\hline Elassoneuria sp. (Ela) \\
\hline Ephemerellidae \\
\hline Ephemerellina sp. (Eph) \\
\hline Plécoptères \\
\hline Perlidae \\
\hline Neoperla sp. (Npe) \\
\hline Coléoptères \\
\hline Elmidae \\
\hline Helminthocaris sp. (Hel) \\
\hline Stenelmis sp. (Ste) \\
\hline Dytiscidae \\
\hline Canthydrus sp. (Can) \\
\hline Rhantaticus sp. (Rhan) \\
\hline Gyrinidae \\
\hline Hydrochidae \\
\hline Hydrochus sp. (Hyd) \\
\hline Hydrophilidae \\
\hline Amphiops sp. (Amp) \\
\hline Enochrus sp. (Eno) \\
\hline Neohydrophilus sp. (Neo) \\
\hline Spercheidae \\
\hline Spercheus sp. (Spe) \\
\hline Curculionidae \\
\hline Trichoptères \\
\hline Polycentropodidae \\
\hline Dipseudopsis sp. (Dip) \\
\hline
\end{tabular}


Tableau I. Suite.

Table I : Continued.

\begin{tabular}{|l|}
\hline Ecnomidae \\
\hline Ecnomus sp. (Ec) \\
\hline Hydropsychidae \\
\hline Macronema sp. (Mac) \\
\hline Diptères \\
\hline Ceratopogonidae (Cer) \\
\hline Chironomidae (Chir) \\
\hline Chironominae \\
\hline Chironomini sp.1 (Chir 1) \\
\hline Chironomini sp.2 (Chir 2) \\
\hline Chironomus gr. plumosus (Chi) \\
\hline Cryptochironomini sp. (Cryp) \\
\hline Cryptochironomus sp. 1 (Cryp 1) \\
\hline Cryptochironomus sp. 2 (Cryp 2) \\
\hline Dicrotendipes sp. (Dicr) \\
\hline Glyptotendipes sp. (Glyp) \\
\hline Microchironomus sp. (Mchi) \\
\hline Nilodosis sp. (Nil) \\
\hline Parachironomus sp. (Pchi) \\
\hline Polypedilum spp. 1 (Pol 1) \\
\hline Polypedilum spp.2 (Pol 2) \\
\hline Tanytarsini spp (Tan) \\
\hline Xenochirnomus sp. (Xchi) \\
\hline Tanypodinae \\
\hline Ablabesmyia sp. (Abla) \\
\hline Cf. Procladius sp. (Pro) \\
\hline Clinotanypus sp. (Cli) \\
\hline Micropelopiinae sp. (Mpel) \\
\hline Orthocladiinae \\
\hline Orthocladiinae sp. (Ortho) \\
\hline Culicidae \\
\hline Mansonia sp. (Man) \\
\hline Psychodidae (Psy) \\
\hline Syrphidae (Syr) \\
\hline
\end{tabular}

\begin{tabular}{|l|}
\hline Tabanidae (Tab) \\
\hline Tipulidae (Tip) \\
\hline Lépidoptères \\
\hline Pyralidae (Pyr) \\
\hline Odonates \\
\hline Gomphidae \\
\hline Ictinogomphus ferox(Rambur, 1842) (Ict) \\
\hline Phyllogomphus sp. (Phy) \\
\hline Coenagriidae \\
\hline Ischnura sp. (Isc) \\
\hline Pseudagrion sp. (Pse) \\
\hline Libellulidae (Lib) \\
\hline Cordulidae (Cor) \\
\hline Hétéroptères \\
\hline Corixidae \\
\hline Agraptocorixa sp. (Agr) \\
\hline Micronecta $s p$. (Meu) \\
\hline Naucoridae \\
\hline Naucoris sp. (Nau) \\
\hline Notonectidae \\
\hline Anisops sp. (Ani) \\
\hline Hydrocyrius sp. (Hco) \\
\hline Notonecta sp. (Not) \\
\hline Plea sp. (Ple) \\
\hline Hydrometridae \\
\hline Hydrometra sp. (Hme) \\
\hline Mesoveliidae \\
\hline Mesovelia sp. (Mes) \\
\hline Nepidae \\
\hline Ranatra sp. (Ran) \\
\hline Belostomidae \\
\hline Rhagovelia sp. (Rha) \\
\hline
\end{tabular}




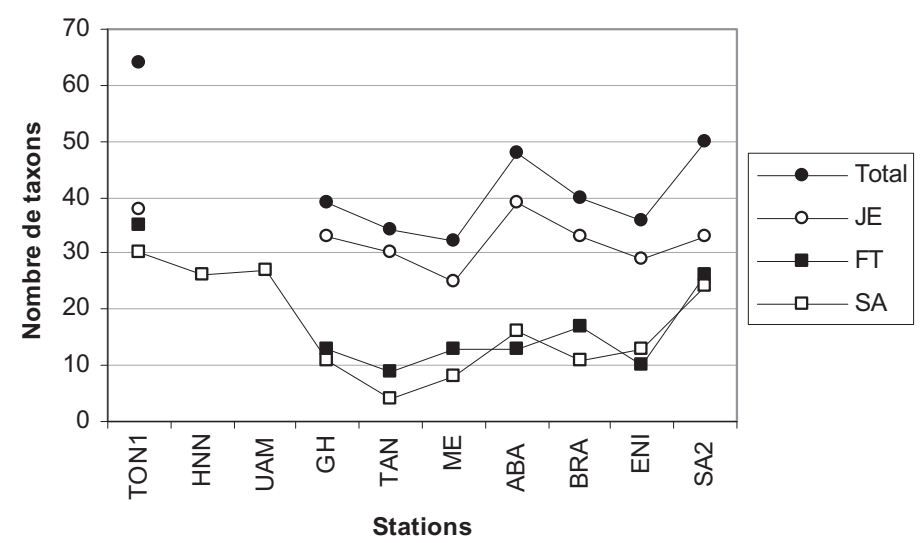

Fig. 2. Variation de la richesse taxonomique des macro-invertébrés du fleuve Niger de l'amont à l'aval des points de rejets de la ville de Niamey sur l'ensemble des prélèvements (JE = jacinthe d'eau, FT = filet troubleau, $\mathrm{SA}=$ substrats artificiels).

Fig. 2. Variation in taxon richness of macroinvertebrates of the River Niger upstream and downstream of the discharge points of Niamey city (JE = Water hyacinth, FT= Net work, SA = artificial substrata).

L'analyse de la distribution longitudinale de la richesse taxonomique totale (Fig. 2) fait ressortir une baisse du nombre de taxons dans les stations en aval immédiat des points de rejets (26 à 48 taxons) comparativement aux stations situées en amont (64 taxons) et en aval (50 taxons) éloigné des rejets.

\section{Étude de la diversité des macro-invertébrés}

La diversité taxonomique sur les substrats artificiels (Fig. 3A) est supérieure à 3 à TON 1 en mai 2005, HNN en juin 2004 et UAM. Elle est comprise entre 1 et 3 à TON 1 en juin 2004, HNN en mai 2005, GH, ME en juin 2004, ABA, BRA en mai 2005 et SA 2. La plus faible diversité taxonomique $(<1)$ est observée à TAN, ME (en mai 2005), ENI et BRA (en juin 2004). Au sein des stations TON 1, HNN, ME et BRA l'indice de diversité est différent pour les deux prélèvements.

La distribution des taxons dans les prélèvements est moyennement équitable à équitable à TON 1, HNN, UAM, $\mathrm{GH}, \mathrm{ME}$ (en juin 2004), ABA (en juin 2004) et SA 2 avec des indices d'équitabilité compris entre 0,52 et 0,92 (Fig. 4A). Elle est inéquitable à TAN, ME (en mai 2005), ABA (en mai 2005), BRA et ENI avec un indice d'équitabilité compris entre 0,1 et 0,39 .

Les prélèvements effectués au filet troubleau donnent des indices de diversité de Shannon-Weaver répartis en trois niveaux (Fig. 3B) : supérieurs à 3 à TON 1 pour tous les prélèvements, inférieurs à 1 à TAN et ME pour le prélèvement de mars 2006 et à ENI pour les prélèvements de juin 2005 et mars 2006 puis compris entre 1 et 3 pour le reste des prélèvements. Ces indices de diversité sont assez similaires à 


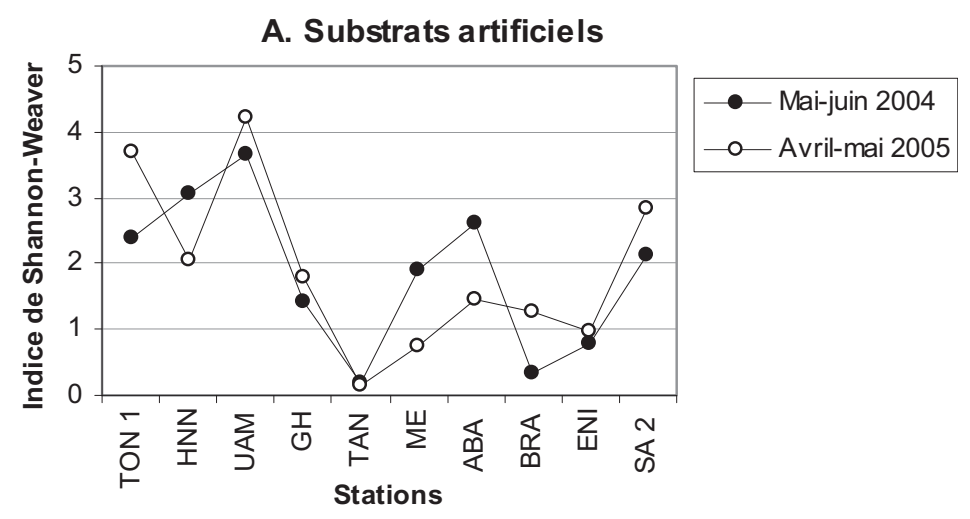

B. Filet troubleau

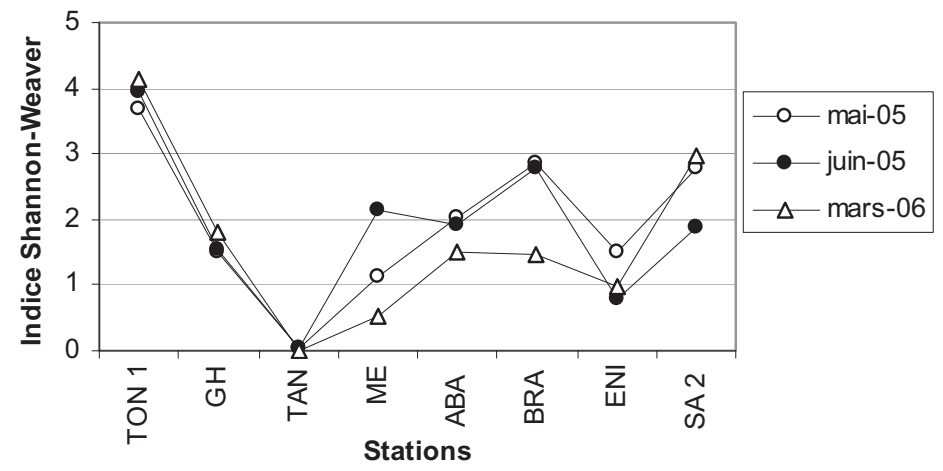

C. Jacinthe d'eau

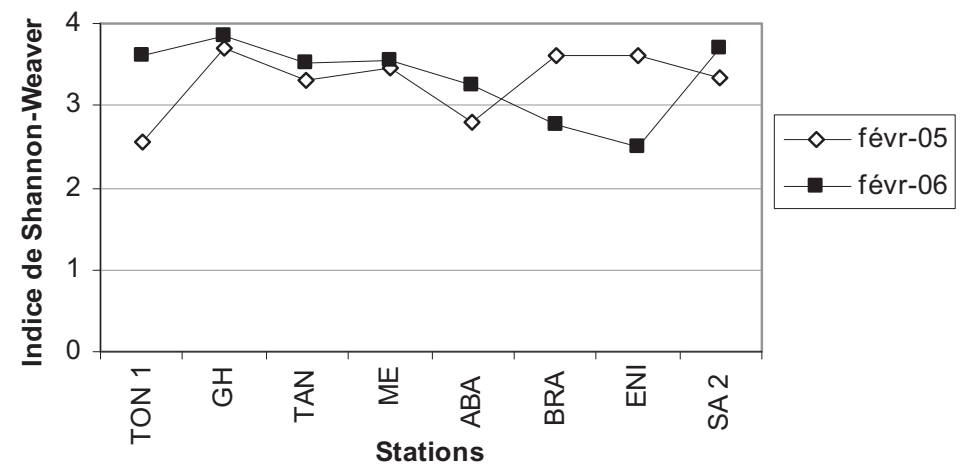

Fig. 3. Variation de la diversité taxonomique de macro-invertébrés du fleuve Niger par technique d'échantillonnage et par prélèvement effectué de l'amont à l'aval des points de rejets de la ville de Niamey.

Fig. 3. Variation of the taxonomic diversity of macroinvertebrates in the River Niger by sampling technique and sampling conducted upstream and downstream of the discharge points of the city of Niamey. 


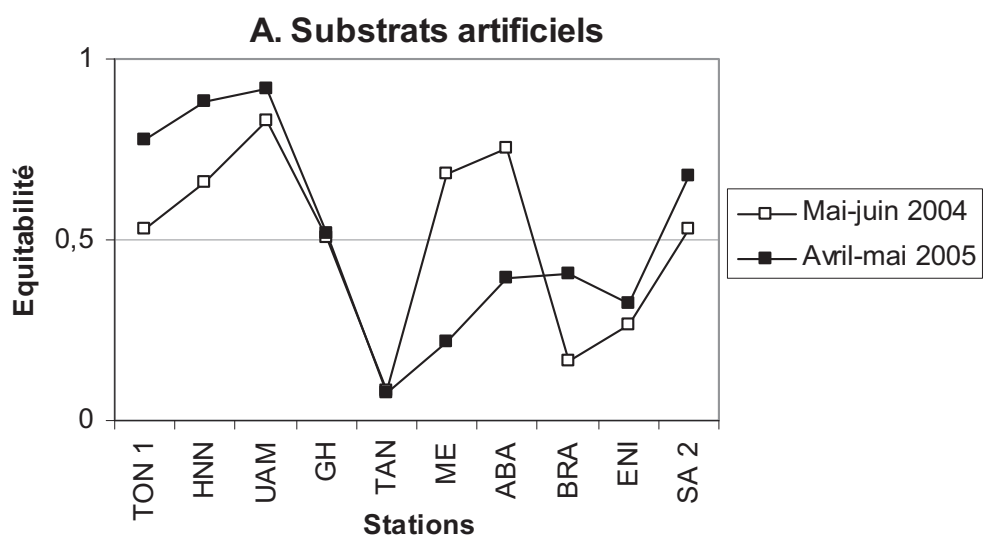

\section{B. Filet troubleau}
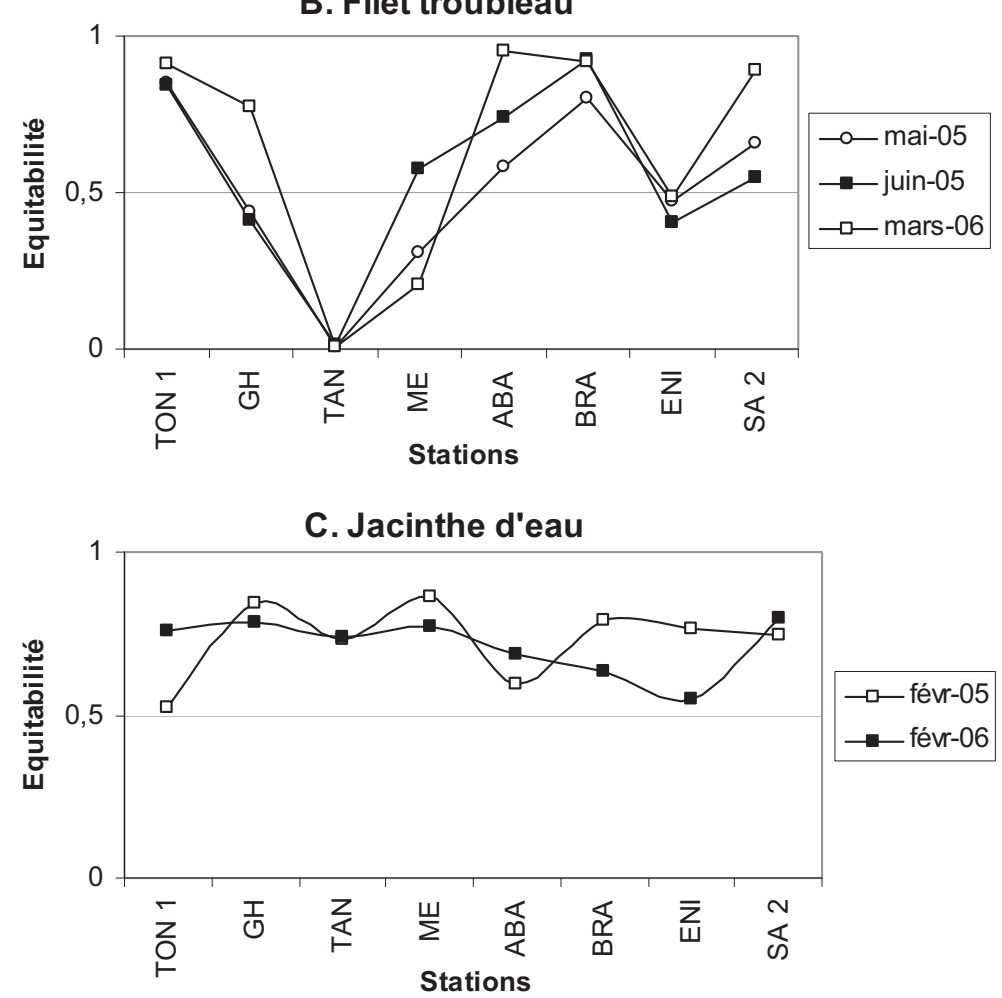

Fig. 4. Variation de l'indice d'équitabilité de Shannon par technique d'échantillonnage de macroinvertébrés et par prélèvement effectué de l'amont à l'aval des points de rejets de la ville de Niamey dans le fleuve Niger.

Fig. 4. Variation of the Shannon equitability index for macroinvertebrates by sampling technique and sample taken upstream and downstream of the discharge points of the city of Niamey in Niger. 
TON 1, GH et TAN. Ils se situent dans le même niveau de qualité des eaux à $A B A, B R A$ et $S A 2$ contrairement à $M E$ et ENI.

L'indice d'équitabilité est très variable d'une station à une autre et souvent entre les prélèvements d'une même station. II reflète une répartition moyennement équitable à équitable des taxons à TON 1, ABA, BRA, GH (en mars 2006), ME (en juin 2005) et SA 2 avec des valeurs comprises entre 0,53 et 0,95 . À GH (en mai et juin 2005) et à ENI, les taxons sont inéquitablement répartis avec des indices situés au tour de 0,40 (Fig. 4B). À ME (en mai 2005 et mars 2006) et TAN, les indices d'équitabilité reflètent une dominance taxonomique avec des valeurs comprises entre 0,1 et 0,30 . Dans l'ensemble, l'indice d'équitabilité est plus élevé en mars 2006 sauf à TAN et ME où Melania sp. est très abondante.

L'indice de diversité de ShannonWeaver calculé sur le système racinaire de la jacinthe d'eau (Fig. 3C) est compris entre 2 et 3 pour les prélèvements effectués aux stations TON 1 et ABA en février 2005 et pour les prélèvements effectués aux stations BRA et ENI en février 2006. Pour le reste des prélèvements, il est compris entre 3 et 4 à toutes les stations. Aux stations TON 1, BRA et ENI, la diversité obtenue en février 2005 et 2006 reflètent deux niveaux différents de qualité des eaux.

L'indice d'équitabilité de Shannon (Fig. $4 C$ ) varie de 0,51 à 0,85 , ce qui indique dans l'ensemble une bonne équitabilité dans la distribution des taxons.

Appliquées à la même période, le filet troubleau et les substrats artificiels donnent des résultats assez semblables sauf à TON 1 et BRA où les substrats artificiels retirés en juin 2004 donnent un indice plus faible compris respectivement entre 2 et 3 puis inférieur à 1 .

Les prélèvements sur la jacinthe d'eau effectués en période de crue donnent les meilleurs indices de diversité dans toutes les stations.

\section{Répartition taxonomique des macro- invertébrés au sein des stations de prélèvements}

L'échantillonnage des macro-invertébrés effectué sur les substrats artificiels permet de mettre en évidence un changement de structure des communautés en passant de l'amont à l'aval des points de rejets de la ville de Niamey grâce à une analyse factorielle de correspondance. Néanmoins, ce changement structurel ou gradient est mal exprimé dans une AFC non illustrée ici à cause de l'influence des stations de la tannerie (TAN) et Mess (ME) caractérisées par une forte dominance de Melania sp.

Dans l'AFC représentée par la figure 5 , les prélèvements effectués dans ces stations sont introduits dans l'analyse en mode passif, ce qui a permis d'aboutir à une distribution des taxons et des stations en forme de croissant dans le plan formé par les axes 1 et 2. Ces derniers expliquent $49 \%$ de la variabilité observée dont $30 \%$ par l'axe 1 et $19 \%$ par l'axe 2 . L'inertie totale est de 2,20.

L'analyse de cette distribution montre à l'extrême gauche la station de référence (TON 1), la station UAM 

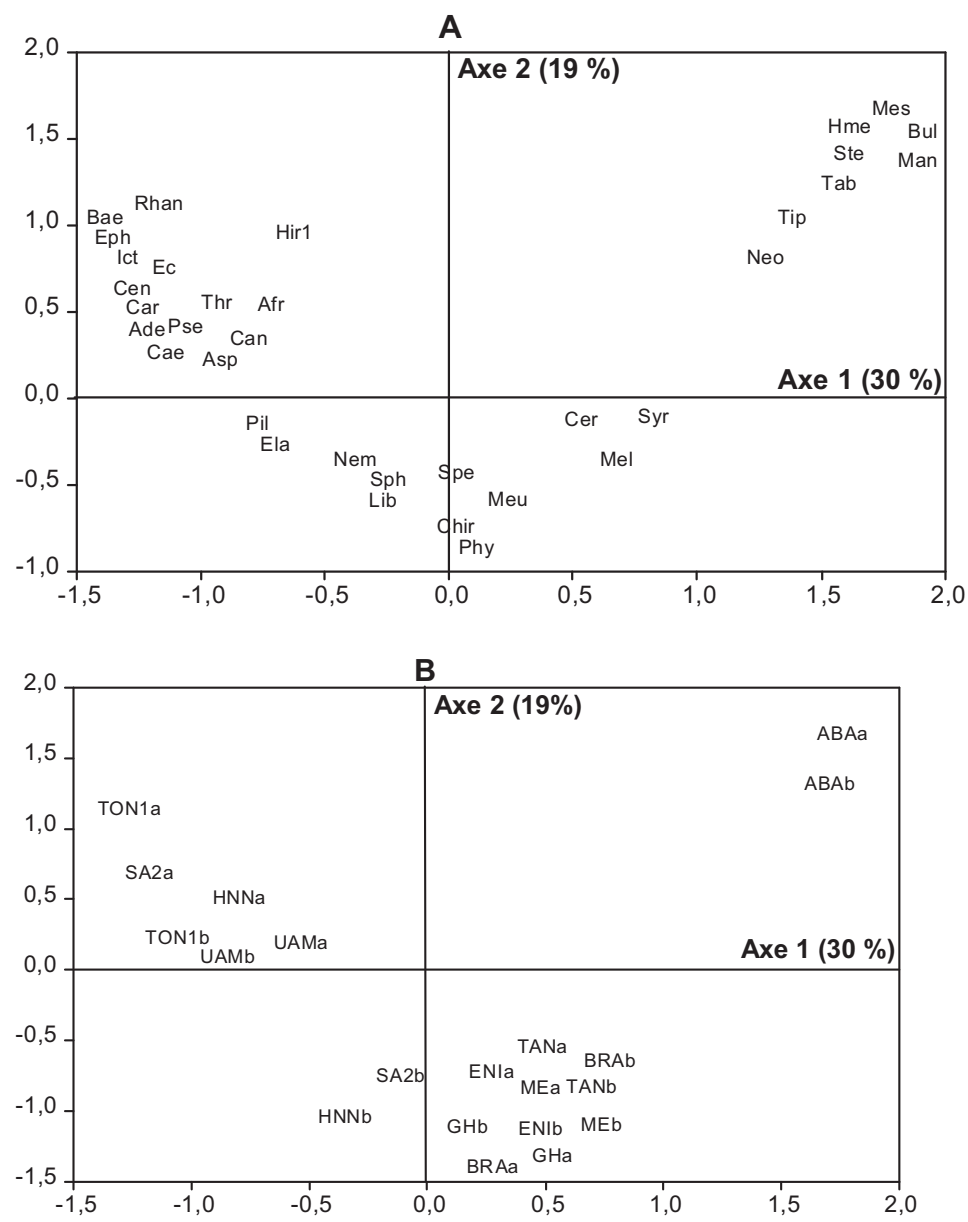

Fig. 5. Ordination des taxons $(A)$ et des prélèvements $(B)$ de macro-invertébrés effectués sur les substrats artificiels de l'amont à l'aval des points de rejets de la ville de Niamey dans le fleuve Niger dans le plan formé par les axes 1 et 2 ( $a=$ mai-juin 2004, $b=$ avril-mai 2005 ; TAN et ME en passif). Fig. 5. Ordination of taxa (A) and samples (B) of macroinvertebrates on artificial substrates made from upstream to downstream discharge points of the city of Niamey in the river Niger in the axis 1 and 2 ( $a$ = May-June 2004, b = April-May 2005, TAN and ME passive).

située en rive droite où les risques de pollution sont faibles, la station HNN qui reçoit les rejets de l'hôpital national de Niamey qui arrivent au fleuve en faible quantité et la station SA 2 située en aval de tous les points de rejets. À cette position des stations correspond un groupe taxonomique composé à
$47 \%$ par les éphéméroptères, $13 \%$ par les trichoptères, $13 \%$ par les coléoptères puis les odonates, les mollusques, les crustacés et les Hirudinés avec $7 \%$ chacun.

À l'extrême droite se trouve la station $A B A$ (rejets de l'abattoir) à laquelle correspond l'ensemble taxonomique 
composé de $38 \%$ de diptères (Tipulidae, Tabanidae et Culicidae), $25 \%$ de coléoptères (Hydrophilidae, Elmidae), $25 \%$ d'hétéroptères (Hydrometridae, Mesoveliidae) et $2 \%$ de mollusques (Bulinidae).

Entre ces deux extrêmes se situent les stations GH, TAN, ME, BRA, ENI et une partie des prélèvements effectués à HNN et SA 2 pour lesquelles correspond un groupe de taxons composé de $25 \%$ de diptères (Chironomidae, Ceratopogonidae et Syrphidae), $25 \%$ de mollusques (Thiaridae, Sphaeridae, Pilidae), $17 \%$ d'odonates (Gomphidae, Libellulidae) puis les coléoptères (Spercheidae), les némathelminthes, Hétéroptères (Corixidae) et les éphéméroptères (Oligoneuriidae) représentant $33 \%$.

L'analyse factorielle de correspondance (AFC) réalisée sur les données de macro-invertébrés recueillies au filet troubleau relate aussi un changement de structure des communautés le long du fleuve Niger à Niamey en passant de l'amont à l'aval des points de rejets de la ville. Elle explique $43 \%$ de la variabilité observée dont $26 \%$ sont expliqués par l'axe 1 et $17 \%$ par l'axe 2, l'inertie totale étant de 2,169. $\mathrm{Ce}$ changement se traduit sur la figure 6A par la distribution le long de l'axe 1 de deux groupes taxonomiques et d'un groupe taxonomique à cheval entre les axes 1 et 2 . Positivement à l'axe 1 est positionné le groupe dominé par des coléoptères ( $23 \%$ ) et des mollusques $(23 \%)$ suivi des odonates $(15 \%)$, des hétéroptères $(15 \%)$, des diptères (15\%) de la famille de Tabanidae et des Syrphidae puis des crustacés de la famille des Atyidae (8\%). Ces taxons au nombre de 13 sont répartis dans les stations $M E, T A N$, $A B A$ et BRA mais rarement présents dans les autres stations (Fig. 6B). À l'opposé de ce groupe, se trouve un ensemble de taxons dominé par les éphéméroptères $(43 \%)$ suivi des trichoptères $(14 \%)$, des coléoptères $(14 \%)$, des plécoptères, des mollusques, des odonates et des crustacés avec chacun $7 \%$. Les 14 taxons de ce groupe sont exclusivement récoltés à TON 1 et SA 2 à l'exception de Caenis $s p$. et de Sphaerium $s p$. présents également à $A B A$. Le dernier groupe est positivement situé sur l'axe 2 et/ou négativement sur l'axe 1. II compte au total 9 taxons. Il est composé des hétéroptères (Micronecta sp. $=$ Meu, Anisops $s p$. = Ani, $22 \%$ ), des Chironomidae (Chir, $22 \%$ ), des mollusques (Pila $s p .=$ Pil), des némathelminthes $(\mathrm{Nem})$, des odonates (Libellulidae $=$ Lib), des trichoptères (Dipseudopsis sp. = Dip) et des éphéméroptères (Cloeon $s p$. = Clo) représentant chacun $11 \%$. Ces taxons se retrouvent dans les stations ENI, GH, TON 1 et SA 2.

La position des prélèvements sur l'axe 1 indique un effet date qui se traduit par un net décalage vers la droite des prélèvements effectués en mars (c). Cet axe met aussi en évidence un effet pollution en séparant les stations situées loin des pollutions des stations localisées en aval immédiat des points de rejets de la ville de Niamey.

L'analyse de la figure 7 résultant de l'AFC sur les abondances de macroinvertébrés récoltés dans le système racinaire de la jacinthe d'eau fait ressortir 6 groupes taxonomiques répartis dans le plan formé par les deux premières composantes (axes 1 et 2). Ces composantes expliquent $42 \%$ de la 

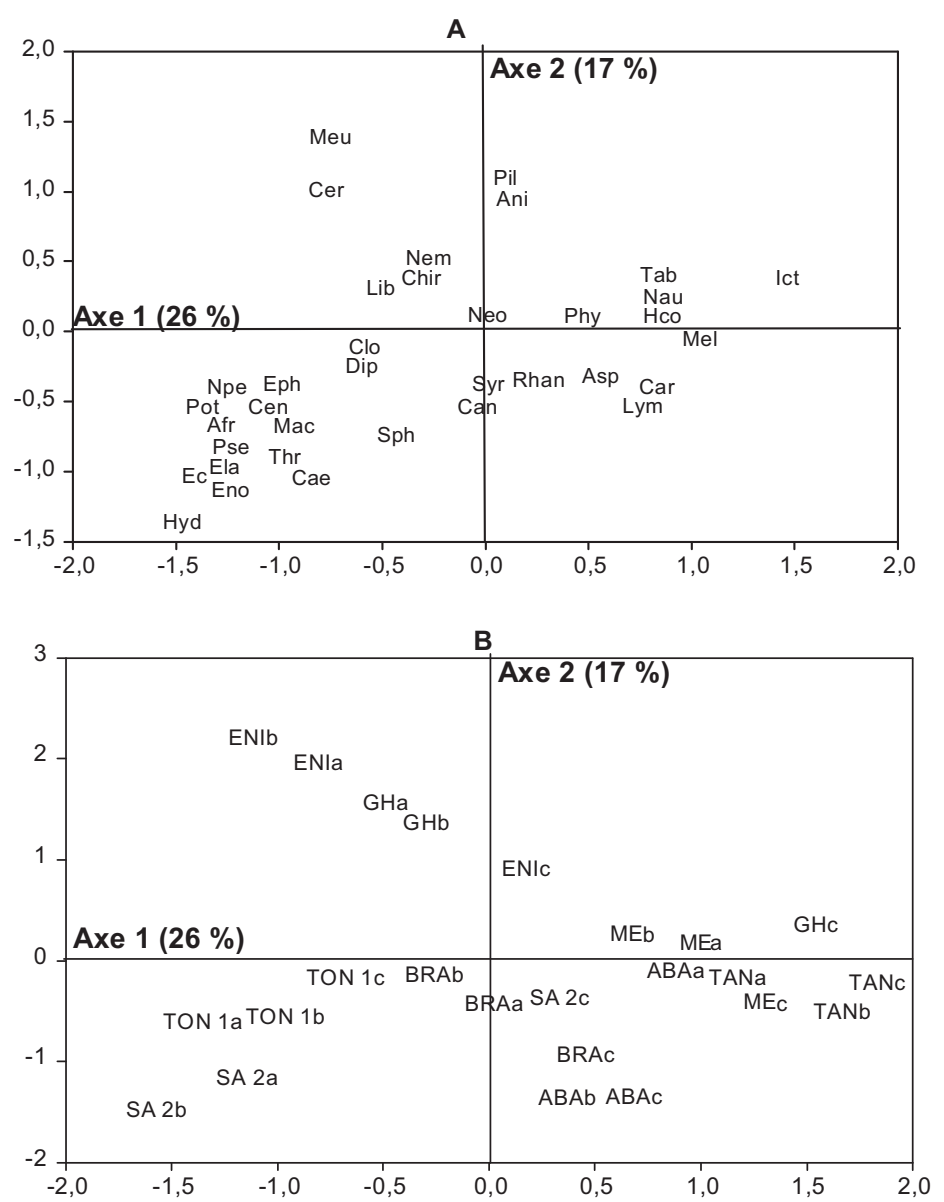

Fig. 6. Ordination des taxons $(A)$ et des prélèvements $(B)$ de macro-invertébrés effectués au filet troubleau de l'amont à l'aval des points de rejets de la ville de Niamey dans le fleuve Niger dans le plan formé par les axes 1 et 2 de l'AFC ( $a=$ mai 2005, $b=$ juin 2005 et $c=$ mars 2006).

Fig. 6. Ordination of taxa $(A)$ and samples $(B)$ of macroinvertebrate collected with the kicknet upstream to downstream discharge points of the city of Niamey in the river Niger in the axis 1 and 2 ( $a=$ May 2005, b = June 2005 and c = March 2006).

variabilité observée dont $25 \%$ sont expliqués par l'axe 1 et $17 \%$ par l'axe 2 . L'inertie totale est de 0,7 . Les taxons composant ces différents groupes présentent une distribution assez large et variée. Ils réduisent la mise en évidence d'un gradient amont-aval.

\section{Classification des stations selon la distance de Bray-Curtis}

La classification des stations, effectuée sur la base de la distance de BrayCurtis calculée dans "R" à partir des valeurs logarithmiques des abondances 

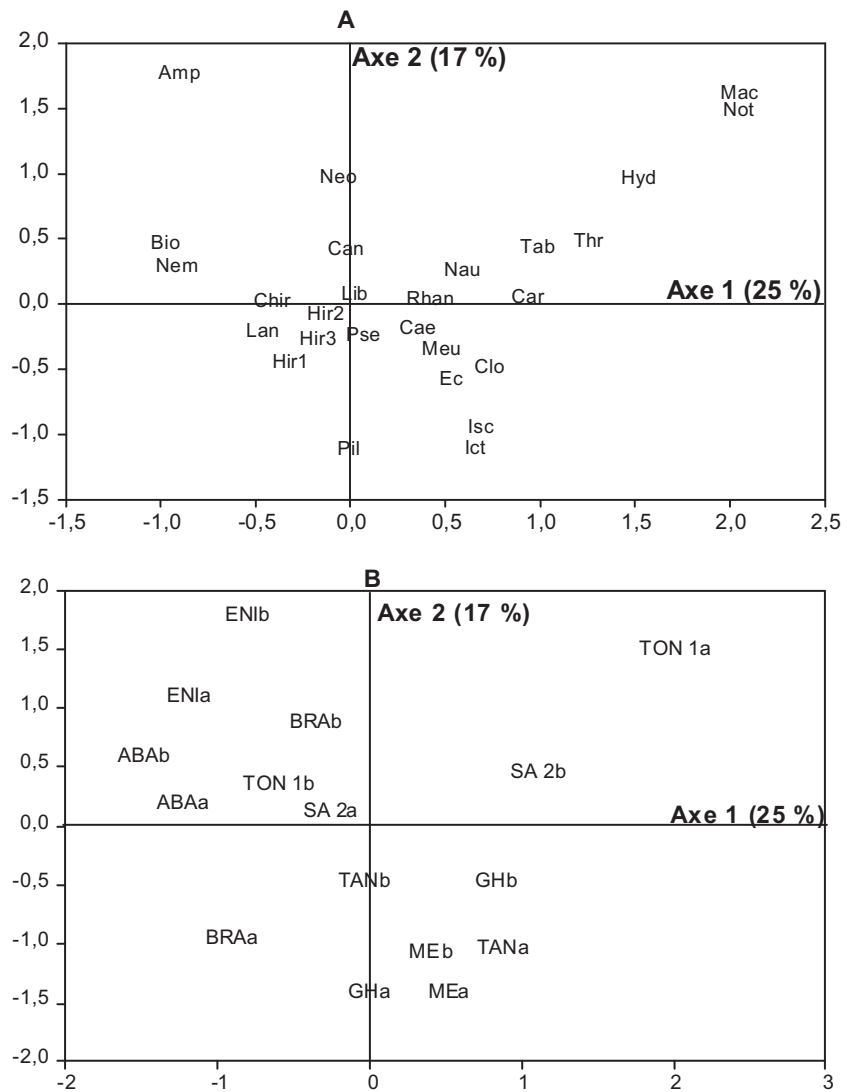

Fig. 7. Ordination des taxons $(A)$ et des prélèvements $(B)$ de macro-invertébrés effectués dans le système racinaire de la jacinthe d'eau de l'amont à l'aval des points de rejets de la ville de Niamey dans le fleuve Niger dans le plan formé par les axes 1 et 2 de l'AFC ( $a=$ février 2005 et $b=$ février 2006). Fig. 7. Ordination of taxa (A) and samples (B) of macroinvertebrates collected in the root system of water hyacinth from upstream to downstream discharge points of the city of Niamey in the river Niger in the axis 1 and 2 of ( $a=$ February 2005 and $b=$ February 2006).

des macro-invertébrés, permet de distinguer trois grands groupes avec les substrats artificiels, quatre grands groupes avec le filet troubleau et deux grands groupes avec la jacinthe d'eau (Fig. 8). Les deux premières méthodes d'échantillonnage (substrats artificiels et filet troubleau) séparent les prélèvements effectués à la station de référence (TON 1) des prélèvements effectués dans les autres stations. Les prélèvements sur la jacinthe d'eau ne permettent pas de ressortir cette différence entre la station TON 1 et les autres stations.

\section{DISCUSSION}

La structure de peuplement des macro-invertébrés le long du fleuve Niger à Niamey est décrite à partir de 

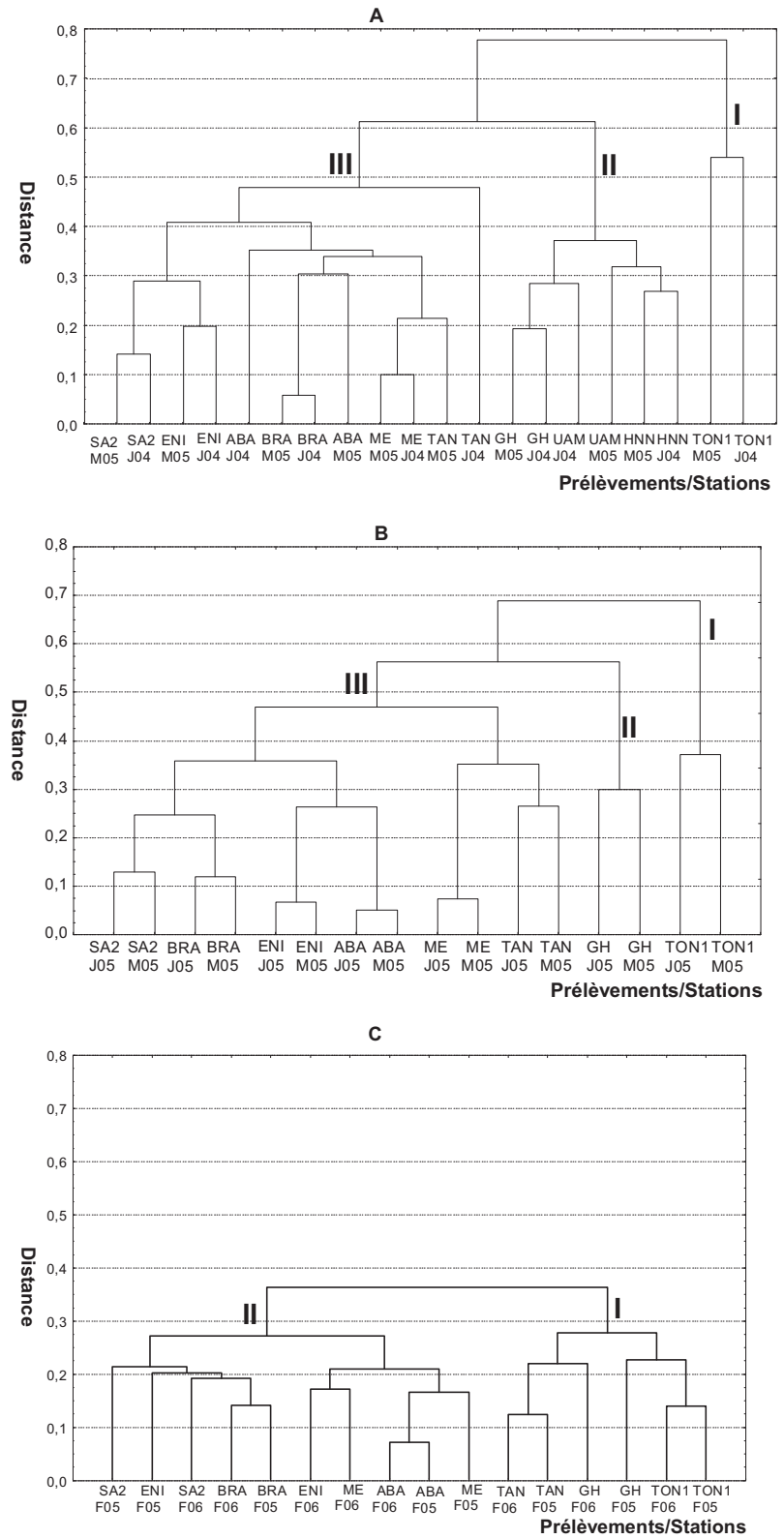

Fig. 8. Classification des stations en fonction de la distance de Bray-Curtis appliquée sur le logarithme des abondances taxonomiques des macro-invertébrés récoltés sur les substrats artificiels $(A)$, au filet troubleau (B) et dans le système racinaire de la jacinthe d'eau (C) ; $M=$ mai, $J=$ juin, $F=$ février.

Fig. 8. Classification of stations based on the Bray-Curtis distance applied to the logarithm of taxonomic abundance of macroinvertebrates collected on artificial substrates $(A)$, the kicknet $(B)$ and in the root system of water hyacinth (C) ; M = May, $\mathrm{J}=$ June, $\mathrm{F}=$ February. 
trois types d'échantillonnage, dont l'applicabilité et la fiabilité sont conditionnées par le régime hydrologique. Deux de ces techniques offrent une meilleure possibilité de comparaison des stations échantillonnées. II s'agit des substrats artificiels et de la jacinthe d'eau contrairement au filet troubleau dont les résultats d'échantillonnage pourraient être influencés par des facteurs comme la diversité d'habitats.

Cette possibilité de comparaison nous amène à expliquer le gradient amont-aval relevé à partir de la richesse et de la diversité taxonomique par l'influence des rejets de la ville de Niamey qui entraînent probablement une altération du milieu. Ainsi, en aval immédiat des rejets, la richesse et la diversité taxonomique connaissent une baisse contrairement à l'amont éloigné des rejets qui représente la situation de référence (Tondibia, située à $7 \mathrm{~km}$ environ en amont de Niamey). En aval lointain, une situation de restauration de la diversité commence à s'observer (SA 2 située à $3 \mathrm{~km}$ environ à l'aval de Niamey). Ces résultats traduisent bien l'évolution d'une altération en passant de l'amont à l'aval du facteur responsable (Rosillon, 1983). Cette altération s'exprime par un nombre plus élevé de taxons en amont des sources de pollution avec une abondance plus faible et l'équitabilité dans la distribution des taxons supérieure à 0,5 contrairement à l'aval immédiat des rejets où le nombre de taxons est plus faible, l'abondance plus élevée et l'équitabilité dans la distribution des taxons inférieure à 0,5 . La richesse et l'abondance taxonomiques sont inversement proportionnelles. Les conditions de milieu défavorables ont donc tendance à favoriser les taxons qui s'adaptent le mieux et prolifèrent au détriment des taxons qui s'adaptent le moins, contrairement à des bonnes conditions de milieu où on tend vers un équilibre entre les abondances taxonomiques.

L'analyse de l'abondance taxonomique montre par exemple une forte dominance de Melania $s p$. à TAN et ME en échantillonnant au filet troubleau et sur les substrats artificiels. Prentice (1983) et Dudgeon (1989) évoquent la capacité de Melania à maintenir une forte densité dans des habitats variés. D'après Chiniotis et al. (1980), Prentice (1983) et Madsen (1992), Melania se nourrit probablement des algues, des micro-organismes et de petites particules de matière organique. La présence à la tannerie (TAN) d'importants dépôts de débris végétaux (Acacia nilotica utilisée dans le tannage des peaux) pourrait justifier la quasi-dominance de Melania dans ce milieu et à la station la plus proche d'elle qui est Mess (ME). À l'abattoir $(A B A)$, malgré une présence abondante de débris végétaux issus des contenus stomacaux, Melania domine uniquement dans les échantillonnages au filet troubleau avec une abondance beaucoup plus faible comparativement à TAN. La proximité de cette station du rejet de l'Office du Lait $\mathrm{du}$ Niger (OLANI) à $\mathrm{pH}$ acide $(\mathrm{pH}$ moyen égal à 5) pourrait être défavorable à la prolifération de ce taxon malgré un $\mathrm{pH}$ compris entre 6 et 7 dans les eaux du fleuve à la station ABA.

L'application des différentes techniques d'échantillonnage à différents régimes du fleuve ne permet pas de les comparer et d'apprécier la performance de chacune d'elle. 
II faut néanmoins noter que ces trois techniques ont permis de se faire une idée sur la richesse taxonomique totale des macro-invertébrés du fleuve Niger à Niamey. La différence de la richesse taxonomique entre les trois techniques montre qu'elles sont complémentaires. En effet, en comparant deux à deux les techniques d'échantillonnage sur la base de la richesse taxonomique, il ressort que les échantillonnages au filet troubleau et sur la jacinthe d'eau ont en commun $49 \%$ des taxons, les substrats artificiels et la jacinthe d'eau $41 \%$, le filet troubleau et les substrats artificiels $56 \%$.

La jacinthe d'eau et les substrats artificiels apportent le plus grand nombre de taxons (72\% du nombre total des taxons chacun) et le filet troubleau $62 \%$. Ces résultats peuvent s'expliquer pour la jacinthe d'eau par l'amélioration des conditions de milieu au moment de l'échantillonnage (crue) effectué en plus dans la colonne d'eau. Quant aux substrats artificiels, ils permettent un échantillonnage plus riche comparé au filet troubleau car ils constituent un micro-habitat supplémentaire induit dans les stations d'échantillonnage et susceptible d'améliorer la diversité taxonomique.

Du point de vue richesse taxonomique, le filet troubleau et les substrats artificiels donnent des résultats plus similaires que ces deux techniques comparées à la jacinthe d'eau. Ces deux techniques, appliquées au même régime du fleuve, échantillonnent le fond et, ils se trouvent de ce fait dans des conditions de milieu comparables.

$\mathrm{La}$ variabilité des abondances taxonomiques observée pour des prélèvements effectués avec une même technique au sein d'une même station pourrait être attribuée aux pertes liées à l'échantillonnage et au tri mais aussi à l'instabilité des eaux dans le lit majeur du fleuve Niger à Niamey qui pourrait être défavorable à l'installation d'une communauté de macro-invertébrés stable. Cela s'observe d'ailleurs à travers la différence de diversité taxonomique entre les prélèvements effectués au filet troubleau dans un mois d'intervalle seulement et la présence des taxons comme Notonecta $s p$. et Ecnomus $s p$. généralement signalés dans des rivières temporaires (Morais, 1995 ; Coimbra et al., 1996) . Le retour progressif du fleuve dans le lit mineur pendant la période d'échantillonnage couplée à une faible diversité d'habitats expliquerait aussi les abondances taxonomiques très faibles observées.

La différence de diversité taxonomique entre les prélèvements effectués sur les substrats artificiels peut être attribuée à des facteurs externes comme le sabotage qui peut perturber le processus de colonisation des substrats par les macro-invertébrés mais aussi à la dynamique de retour des eaux dans le lit mineur. La variation de la diversité taxonomique dans les prélèvements réalisés sur le système racinaire de la jacinthe d'eau est tributaire de l'arrivée de ce macrophyte en saison des pluies, de son processus de stabilisation le long des berges et de sa prolifération. La jacinthe d'eau a une densité non seulement plus élevée dans la zone de rejet qu'en amont et en aval loin des rejets mais variable aussi d'une année à une autre.

La comparaison de la diversité taxonomique apportée par les substrats artificiels et le filet troubleau 
montre des différences entre la station de référence, la station en aval de tous les points de rejets et les stations en aval immédiat des rejets. Ces techniques d'échantillonnage sont appliquées en étiage, période où la dilution des rejets est très faible alors que la jacinthe d'eau est échantillonnée en période de crue pendant que le pouvoir de dilution du fleuve est élevé.

L'analyse factorielle de correspondance appliquée à l'ensemble des macro-invertébrés démontre les mêmes résultats en donnant une distribution des taxons selon un gradient écologique qui serait significativement $(P<$ $0,05)$ expliqué par les matières organiques et oxydables sur la jacinthe d'eau et par les matières azotées, phosphorées, organiques et oxydables lorsque l'échantillonnage est effectué au filet troubleau.

Les macro-invertébrés montrent à travers l'AFC une différence entre les stations de référence et les stations situées dans les zones de rejets. La comparaison des trois AFC sur la base de l'inertie totale expliquée privilégie l'utilisation des substrats artificiels et du filet troubleau qui présentent respectivement 2,20 et 2,17 d'inertie totale. Avec la jacinthe d'eau, l'inertie expliquée est de 0,7 , ce qui traduit moins la variabilité entre les stations conformément à la période d'échantillonnage. Ces résultats montrent en plus que la pollution des eaux du fleuve Niger à Niamey est beaucoup plus perceptible en étiage qu'en période de crue.

L'utilisation de la méthode de BrayCurtis pour la classification des stations traduit le même phénomène. La distance d'agrégation des stations, qui se fait entre 0 et 0,8 pour les substrats artificiels et le filet troubleau puis entre 0 et 0,4 pour la jacinthe d'eau, montre bien que dans le premier cas (substrats artificiels et filet troubleau) les stations de prélèvement sont beaucoup plus différentes les unes des autres que dans le second cas (jacinthe d'eau).

L'analyse de l'évolution des taxons de la station de référence en passant par les zones de rejets puis par les stations en aval éloigné des rejets montre que les taxons comme Baetis sp., Ephemerellina sp., Centroptiloides sp., Thraulus sp., Afronurus sp., Adenophlebia sp., Caenis sp., Elassoneuria $s p$. et Ecnomus sp. sont absents ou deviennent rares dans la zone de rejets pour réapparaître dans les stations en aval éloigné des rejets. Dans les stations en aval immédiat des rejets, apparaissent des taxons beaucoup plus tolérants à certaines conditions du milieu comme un déficit en oxygène. Ces taxons appartiennent par exemple aux Chironomidae, Culicidae, Syrphidae (Coimbra et al., 1996). Une telle évolution des taxons est une indication sur la différence des caractéristiques des stations échantillonnées qui peut s'expliquer non seulement par la différence d'habitats mais aussi par l'impact des rejets sur le milieu récepteur. II faut en effet noter que la diversité d'habitats est plus importante à la station de référence et à la station en aval éloigné des rejets du fait de la présence de pierres ou cailloux absents sur toute la zone de rejets.

En plus, la présence chez Chironomus gr. plumosus de tubules assez développées dans toutes les stations en aval des rejets pourrait être considérée comme une indication de pollution 
organique. Cette espèce n'a jamais été récoltée à la station de référence, TON 1, ce qui pourrait être une indication d'une absence entre autres de pollution organique à cette station.

\section{RÉFÉRENCES BIBLIOGRAPHIQUES}

Angelier E., 2000. Ecologie des eaux courantes. Editions Technique \& Documentation, $199 \mathrm{p}$.

Bauernfeind E. \& Moog O., 2000. Mayflies (Insecta: Ephemeroptera) and the assessment of ecological integrity: a methodological approach. Hydrobiologia 422/423 : 71-83.

Chiniotis B.N.C., Butler J.M., Ferguson F. Jr. \& Jobin W.R., 1980. Bionomics of Tarebia Granifera (Gastropoda: Thiaridae) in Puerto Rico, an Asiatic vector of Paragonimiasis Westermani. Caribbean Journal of Science 16 (1-4) : 81-90.

Coimbra C.N., Graça M.A.S. \& Cortes R.M., 1996. The effects of a basic effluent on macroinvertebrate community structure in a temporary Mediterranean river. Environmental Pollution 94 : 301-307.

Dejoux C., 1991. Les macro-invertébrés associés à la végétation aquatique dans la partie bolivienne du lac Titica. Revue Hydrobiologie Tropicale 24 : 91-104.

Dudgeon D., 1989. Ecological strategies of Hong Kong Thiaridae (Gastropoda: Prosobranchia). Malacological Review 22 : 39-53.

Durand J.R. \& Leveque C., 1980. Flore et faune aquatiques de l'Afrique sahelosoudanienne. Tome I. ORSTOM. Paris, $389 \mathrm{p}$.

Durand J.R. \& Leveque C., 1981. Flore et faune aquatiques de l'Afrique sahelosoudanienne. Tome II. ORSTOM. Paris, $847 \mathrm{p}$.
Evrard M., 1996. Utilisation des exuvies nymphales de Chironomidae (Diptera) en tant qu'indicateurs biologiques de la qualité des eaux de surface wallonnes. Thèse de Doctorat, Facultés Universitaires Notre Dame de la Paix Namur (Belgique), 204 p. (sans les annexes).

Floury M., Usseglio-Polatera P., Ferreol M., Delattre C. \& Souchon Y., 2013. Global climate change in large European rivers: long-term effects on macroinvertebrate communities and potential local confounding factors. Global Change Biology 19 : 1085-1099.

Gayraud S., Statzner B., Bady P., Haybachp A., Schôll F., Usseglio-Polatera P. \& Bacchi M., 2003. Invertebrate traits for the biomonitoring of large European rivers: an initial assessment of alternative metrics. Freshwater Biology 48 : 20452064.

Lafont M., Juget J. \& Rofes G., 1991. Un indice biologique lacustre basé sur l'examen des oligochètes. Revue des sciences de l'eau 4 : 253-268.

Lancaster J., 1999. Small-scale movements of lotic macroinvertebrates with variations in flow. Freshwater Biology 41 : 605-619.

Legendre P. \& Legendre L., 1998. Numerical Ecology. Second English edition. Elsevier Science B.V., Amsterdam, $853 \mathrm{p}$.

Lomond T.M. \& Colbo M.H., 2000. Variations in lake-outlet Ephemeroptera, Plecoptera, and Trichoptera communities amongst regions of eastern Newfoundland, Canada. Canadian Journal of Zoology 78 : 1536-1543.

Madsen H., 1992. Food selection by freshwater snails in the Gezira irrigation canals, Sudan. Hydrobiologia 228 : 203-217.

Mondy C.P., Villeneuve B., Archaimbault V. \& Usseglio-Polatera P., 2012. A new 
macroinvertebrate-based multimetric index (I2M2) to evaluate ecological quality of French wadeable streams fulfilling the WFD demands: A taxonomical and trait approach. Ecological Indicators $18: 452-467$.

Morais M.M., 1995. Organização especial e temporal de um rio temporário mediterrânico (Rio Degebe, bacia hidrográfica do Guadiana). Descritores fisico-quími$\cos$ e produtores primarios. Comunidades bentónicas de macroinvertebrados. PhD Thesis. University of Évora, Évora, Portugal. Coimbra C.N., Graça M.A.S., Cortes R.M., 1996. The effects of a basic effluent on macroinvertebrate community structure in a temporary Mediterranean river. Environmental Pollution 94 : 301-307.

Muli J.R. \& Mavuti K.M., 2001. The benthic macrofauna community of Kenyan waters of Lake Victoria. Hydrobiologia $458: 83-90$.

Pelletier L., 2002. Le bassin de la rivière Saint-Maurice : les communautés benthiques et l'intégrité biotique du milieu. Québec, ministère de l'environnement, direction du suivi de l'état de l'environnement, envirodoq $n^{\circ} E N V / 2002 / 0291$, rapport $n^{\circ} E A / 2002-02,85 p$.

Piscart C., Moreteau J.C. \& Beisel J.N., 2005. Biodiversity and structure of macroinvertebrate communities along a small permanent salinity gradient (Meurthe River, France). Hydrobiologia, $551: 227-236$.

Prentice M.A., 1983. Displacement of Biomphalaria glabrata by the snail Thiara granifera in field habitats in Santa Lucia, West Indies. Annals of tropical medicine and parasitology 77 (1) : 51-59.

Quinn J.M. \& Hickey C.W., 1990. Characterization and classification of benthic invertebrate communities in $88 \mathrm{New}$ Zealand rivers in relation to environmental factors. New Zealand Journal of
Marine and Freshwater Research 24 : 387-407.

R Development Core Team, 2007. R: A language and environment for statistical computing. R Foundation for Statistical Computing, Vienna, Austria.

Rosillon D., 1983. Étude quantitative de l'impact d'une pollution organique sur la biocénose benthique d'une rivière salmonicole : La Lhomme (Belgique). Annales de la Societé Royale de Zoologie de Belgique 1 : 19-30.

Sarkar S.K., Bhattacharya A., Giri S., Bhattacharya B., Sarkar D., Nayak D.C. \& Chattopadhaya A.K., 2005. Spatiotemporal variation in benthic polychaetes (Annelida) and relationships with environnemental variables in a tropical estuary. Wetlands Ecology and Management 13 : 55-67.

Shivoga W.A., 2001. The influence of hydrology on the structure of invertebrate communities in two streams flowing into Lake Nakuru, Kenya. Hydrobiologia $458: 121-130$.

Solimini A.G., Gulia P., Monfrinotti \& Carchini G., 2000. Performance of different biotic indices and sampling methods in assessing water quality in the lowland stretch of the Tiber River. Hydrobiologia 422/423 : 197-208.

Statsoft France, 1997. Statistica pour Windows (manuel du programme): Statsoft France - 31 cours des Juilliottes - 94700 Maisons-Alfort.

Statzner B., Doledec S. \& Hugueny B., 2004. Biological trait composition of European stream invertebrate communities: assessing the effects of various trait filter types. Ecography 27 : 470-488.

Ter Braak C.J.F. \& Smilauer P., 1999. CANOCO for Windows (version 4.02) a FORTRAN program for canonical community ordination. Centre for 
biometry Wageningen. Wageningen. The Netherlands.

Tumwesigye C., Yusul S.K. \& Makanga B., 2000. Structure and composition of benthic macroinvertebrates of a tropical forest stream, River Nyamweru, western Uganda. African Journal of Ecology 38 : 72-77.

Usseglio-Polatera P., Bournaud M., Richoux P. \& Tachet H., 2000. Biomonitoring through biological traits of benthic macroinvertebrates: how to use species trait databases? Hydrobiologia 422/423 : 153-162.
Vanden Bossche J.P.\& Usseglio-PolateraP. 2005. Characterization, ecological status and type-specific reference conditions of surface water bodies in Wallonia (Belgium) using biocenotic metrics based on benthic invertebrate communities. Hydrobiologia 551 : 253-271.

Viljoen A., Cyrus D.P. \& Wepener V., 2001. Comparison of the density and species composition of aquatic invertebrates found between the roots of Eichhornia crassipes plants from two coastal lakes in northern KwaZulu-Natal. African Journal of Aquatic Science 26 : 57-65. 